with a catchment area of 500,000 people, one Advanced and 4 Basic Life Support teams. We used a qualitative approach and interviewed providers. Inclusion criteria were: healthcare professionals working with SAMU or the regional trauma hospital Metropolitano for at least 6 months. Interviews focused on primary causes and measures to reduce delays in care of RTI patients.

Results We interviewed 11 providers: 2 physicians and 2 nurses from Metropolitano and 7 SAMU employees (1 nurse, 3 physicians, 3 drivers). Primary causes of delays fell into the following categories: 1) lack of public education, 2) traffic, 3) insufficient personnel/ambulances, 4) bureaucracy, and 5) poor location of stations. Traffic was the most common response, with a total of 7 responses, including nearly all SAMU providers. Suggested measures to reduce delays were: 1) improving public education, 2) increasing personnel, 3) increasing ambulances, 4) proper extrication/need for rapid treatment, and 5) need for a centralised station to avoid traffic. The most common response was the need for public education, primarily teaching drivers about ambulance right-of-way.

Conclusions Most providers, particularly SAMU providers, believe traffic is the primary cause of delay in presentation of RTI patients to a tertiary care centre. Rapid economic growth and increased road traffic are primary factors leading to the overall increased rates of RTIs in LMICs. The same traffic causing RTIs may also be a significant cause of delay in the treatment these patients. Offered solutions to reduce delays focused mostly on public education and the need for increased resources. A public education campaign for driver education in response to RTIs could be an initial step towards reducing delays in the care of RTI patients.

\section{THE AGREEMENT OF TRIAGE RESULT BY REGISTERED NURSE AT DISPATCH CENTRE, ON SCENE AND EMERGENCY ROOM}

${ }^{1}$ Daoruang Kommuangpuk, ${ }^{2}$ Anuchar Sethasathien. ' Udonthani Hospital, Thailand; ${ }^{2}$ National Institute for Emergency Medicine, Thailand

\subsection{6/injuryprev-2016-042156.591}

Background The $8^{\text {th }}$ regional health area of Thailand, have 7 province and 88 hospitals. Three phase triage, first at dispatch centre second on scene and third in emergency room (ER) were done by difference registered nurses (RN). One patient will be 3 times triage by 3 difference $\mathrm{RN}$.

Methods This Quasi-experimental study focus on agreement of triage result by RN from 88 hospitals, compared between before and after triage training. RN was practically trained, triage competency development for modified Emergency Severity Index (ESI), on June 2014. The data was collected on May and July 2014. Analysed by kappa statistics and 95\% CI: of kappa. The percent agreement before and after training triage was compared by Chi-square test.

Results 3,325 patients were triaged, almost triage level were yellow and red. There were 24 Clinical-based categories of Emergency service. Top three of the Categories were Motor Vehicle Accident (22.20\%) Sick (17.75\%) Unconscious (8.30\%). Before training, the agreement between dispatch and scene was almost perfect with kappa 0.82 (95\% CI: $=0.78-0.87)$ the agreement between scene and ER was moderate with kappa 0.47 (95\% CI: $=0.42-0.52)$. After training, the agreement between dispatch and scene was almost perfect with kappa 0.85 (95\% CI: $=0.80-$
0.89) the agreement between scene and ER was moderate with kappa 0.57 (95\% CI: $=0.52-0.61)$. The percent agreement of dispatch and scene triage between before and after training were not different. But the percent agreement of scene and ER triage were significantly improved (P-value $=0.001$ ), with 0.05 level of significance.

Conclusions The agreement of scene and ER triage was moderate, since patients were received first aid and treatment in transit that did change for level of triage when they came to ER. But after training, this agreement was better than before. This study confirm that we can improve triage agreement for $\mathrm{RN}$ by triage training and we should improve the other first aid and treatment competency for RN.

\section{INJURED PATIENTS' INTERACTIONS WITH HEALTH PROVIDERS: IMPLICATIONS FOR ENHANCING TRAUMA CARE}

${ }^{1}$ Sandy Braaf, ${ }^{2}$ Shanthi Ameratunga, ${ }^{3}$ Nicola Christie, ${ }^{1}$ Peter Cameron, ${ }^{4}$ Ronan Lyons, ${ }^{5}$ James Harrison, ${ }^{1}$ Jennie Ponsford, ${ }^{1}$ Alex Collie, ${ }^{6}$ Mark Fitzgerald, ${ }^{7}$ Rodney Judson, ${ }^{8}$ Andrew Nunn, ${ }^{9}$ David Attwood, ${ }^{10}$ Helen Jowett, ${ }^{10}$ Warwick Teague, ${ }^{1}$ Belinda Gabbe. ${ }^{1}$ Monash University, Australia; ${ }^{2}$ University of Auckland, New Zealand; ${ }^{3}$ University College London, United Kingdom; ${ }^{4}$ Swansea University, United Kingdom; ; ${ }^{5}$ Flinders University, Australia; ${ }^{6}$ The Alfred, Australia; ${ }^{7}$ Royal Melbourne Hospital, Australia; ${ }^{8}$ Victorian Spinal Cord Service, Australia; ${ }^{9}$ Transport Accident Commission, Australia; ${ }^{10}$ Royal Children's Hospital, Australia

\subsection{6/injuryprev-2016-042156.592}

Background The path to recovery following major trauma can involve a long trajectory of complex health care needs and multiple interactions with health professions. We explored the perspectives of seriously injured patients regarding issues that arise during their interactions with health providers.

Methods This qualitative study, nested within a population-based longitudinal cohort study, involved semi-structured telephone interviews conducted three years following injury with 64 adult major trauma patients purposively sampled from the Victorian State Trauma Registry. We report one aspect here. Thematic analysis was undertaken of interview transcripts.

Results The importance of effective communication was a theme that had implications in the in-hospital, rehabilitation and community care settings. Effective communication occurred when service providers conveyed detailed information in a sensitive and clear manner, frequently and actively involved patients in discussion, and were responsive to patients' questions. Such interactions encouraged information exchange and shared decisionmaking. Ineffective communication arose predominately during in-hospital care and at discharge from inpatient facilities. Themes related to hospital care included limited contact with health professionals, inability to process information, indirect communication, and struggling to deal with multiple health professionals. At hospital and rehabilitation discharge, themes included insufficient patient engagement, inadequate information flow and feeling disregarded. Ineffective communication resulted in discontinuity of care and preventable health problems.

Conclusions The communication and information needs of seriously injured patients were inconsistently met over the course of their recovery. The findings reveal the need for trauma care systems to support relevant training of service providers, engage patients in planning decisions, and provide information in appropriate forms. 